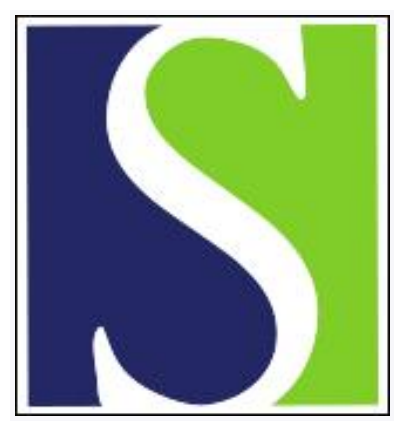

Scand J Work Environ Health 1987;13(4):352-355

https://doi.org/10.5271/sjweh.2029

Issue date: Aug 1987

Thermographic assessment of skin temperature during a cold provocation test.

by Dupuis $\mathrm{H}$

Affiliation: Institute of Occupational Health and Social Medicine, Johannes Gutenberg University, Mainz, Federal Republic of Germany.

This article in PubMed: www.ncbi.nlm.nih.gov/pubmed/3433037

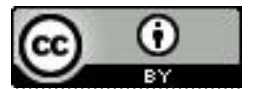




\title{
Thermographic assessment of skin temperature during a cold provocation test
}

\author{
by Heinrich Dupuis, Prof $\mathrm{Dr}^{1}$
}

\begin{abstract}
DUPUIS H. Thermographic assessment of skin temperature during a cold provocation test. Scand $J$ Work Environ Health (13) (1987) 352-355. A description was made of the development and application of infrared thermography and fingertip thermometry for the assessment of finger skin temperature during a cold provocation test. Three hundred and seventeen lumberjacks, grinders, metalworkers, stone cutters, and students were examined in laboratory and field investigations. Under laboratory conditions, the influence of water temperature and exposure duration was studied, and skin temperature measurements were made on the volar and dorsal side of the fingers. In the field occupational health examination, infrared thermography and fingertip thermometry were simultaneously applied during a cold provocation test. The following three types of temperature reaction could be recognized: normal rewarming and moderate delay and strong delay of rewarming. The temperature distribution along the finger length was analyzed. The following conclusions were drawn for practical application of the thermometric methods. Skin temperature must be measured on all 10 fingertips, either on the volar or on the dorsal surface. Fingertip thermometry may be used in occupational health examinations. Infrared thermography may yield more information on the development of disturbances in peripheral circulation along the finger length and may be used in special clinical work.
\end{abstract}

Key terms: fingertip thermometry, infrared thermography, thermoregulation, vibration-induced white finger.

Since 1976 in Germany, vibration-induced white finger (VWF) has been included in the official list of occupational diseases, but there are yet no guidelines for the diagnostic methods to be used for this disorder. The Ministry of Research and Technology and the Central Association of the Industrial Injuries Insurance Institutes have financed research with the purpose of establishing diagnostic methods developed under laboratory conditions and tested in the field. However, for several reasons, there has been no attempt to obtain epidemiologically valid data. The results of this project will be used for the standardization of screening tests in occupational health examinations of vibrationexposed workers. The part of the project described in the present report concerns fingertip thermometry and infrared thermography on subjects with and without Raynaud's phenomenon.

\section{Subjects and methods}

The investigation comprised a total of 317 subjects. Seventyfive of them were students [mean age 25.2 (SD 3.8) years], 10 were staff members of the Institute [mean age 29.2 (range 20-36) years], and 15 were lumberjacks [mean age 38.0 (SD

\footnotetext{
1 Institute of Occupational Health and Social Medicine, Johannes Gutenberg University, Mainz, Federal Republic of Germany.
}

Reprint requests to: Prof $\mathrm{Dr} \mathrm{H}$ Dupuis, Institut für Arbeitsund Sozialmedizin, Hüffelsheimerstr 5, D-6550 Bad Kreuznach, FRG.
13.7) years] examined in the preliminary clinical study. Eightyfour lumberjacks [mean age 37.6 (SD 9.5) years], 65 grinders [mean age $34.5(\mathrm{SD}, 9.6)$ years], 62 metalworkers [mean age 37.9 (SD 8.5) years], and 6 stone cutters [mean age 40.3 (range 21-53) years] were studied in connection with the field occupational health examination. These workers were employed in steel, automobile, and brown-coal industries and in forestry. The total average vibration exposure time was $5188 \mathrm{~h}$ for the lumberjacks and $13329 \mathrm{~h}$ for the grinders, the times for the metalworkers and stone cutters varying greatly. Considering the heterogeneity in age, exposure time, and environmental factors of the study group and the smal! number of subjects, it was not appropriate (and not intended) to use the collected data for any epidemiologic research purposes.

In addition to other methods (general health examination, occupational history, anamneses on peripheral circulation and nervous function disturbances, nerve conduction velocity, vibration perception threshold, and temporary threshold shift) reported elsewhere (1), a cold provocation test was carried out, together with infrared thermography, and fingertip thermometry.

In a pilot study it was shown that water temperatures below $10^{\circ} \mathrm{C}$ gave rise to pain and therefore were not generally tolerated. A few subjects were excluded because of circulatory debility. At water temperatures above $10^{\circ} \mathrm{C}$, a variation of $2^{\circ} \mathrm{C}$ did not influence the thermal reaction of the skin during the test. Doubling the time of hand immersion in the water led, however, to a prolongation of the rewarming time. For practical reasons it was desirable that the duration of the complete test not exceed $30 \mathrm{~min}$. Therefore, in the field research, the conditions of the cold provocation test were standardized as follows: room temperature $22-24^{\circ} \mathrm{C}$, acclimatization before the test $20 \mathrm{~min}$, water temperature $15^{\circ} \mathrm{C}$, immersion time $1 \mathrm{~min}$, test duration $30 \mathrm{~min}$.

The following two methods for measuring skin temperature were used simultaneously in all cases:

1. The skin temperature was measured during the test from a thermosensor attached to the volar surface of each finger- 
tip. The temperature was measured every $12 \mathrm{~s}$, stored, computed, and recorded on a 6 -color plotter. The fingertip thermometry system is shown in figure 1 .

2. In order to obtain information on skin temperature not only at one point, but along the finger length and the hands, infrared thermography was also used. By way of temperature radiation, the system (as shown in figure 2) enables the recording of temperature images of the dorsal sides of both hands. Thus, during the test, 16 images could be recorded and stored in a computer. Special software allowed the analysis of temperature profiles along the finger and the drawing of rewarming curves for the measuring points on the fingertips (in figure 5 of the Results and Discussion section).

\section{Results and discussion}

The temperature measurements made on 90 fingers in a pilot study showed small differences between the volar and dorsal surfaces (mean $0.2^{\circ} \mathrm{C}$, maximum $0.9^{\circ} \mathrm{C}$ ). The results of the two thermometric methods did not differ very much in accuracy and always showed the same trend. The values of the fingertip thermometry sometimes tended to be lower than those measured with the infrared thermography, the reason possibly being the difficulty in thoroughly drying the skin of the area around the thermosensors after the cold provocation.

There is no absolute and generally agreed measure of "normal" and "pathological" reactions to guide the choice of the rewarming criterion. In principle it is, however, clear that fast rewarming is a sign of high dynamic activity of the muscles of the blood vessels, whereas delayed recovery is a symptom of weak dynamic activity. Different criteria may be used to follow this principle.

The background for the choice made in the present work was the following. Temperature recovery for healthy persons mostly showed exponential rewarming curves, ie, normally recovering peripheral vessels dilate some minutes after the end of the cold provocation.
Thus the skin temperature at the fingertips increases rapidly up to about $28^{\circ} \mathrm{C}$ or higher (curve 1 in figure 3). Further increase in skin temperature becomes continuously smaller and smaller however. Delayed recovery may be characterized by a temperature of $<28^{\circ} \mathrm{C}$ after $15 \mathrm{~min}$ (curves 2,3 , and 4 in figure 3 ). In this way the analysis of temperature recovery of 2220 fingers of 222 subjects (lumberjacks, grinders, metalworkers, students) revealed the following three reaction types:

Normal recovery: Fingertip skin temperature of $\geq 28^{\circ} \mathrm{C}$ within $15 \mathrm{~min}$

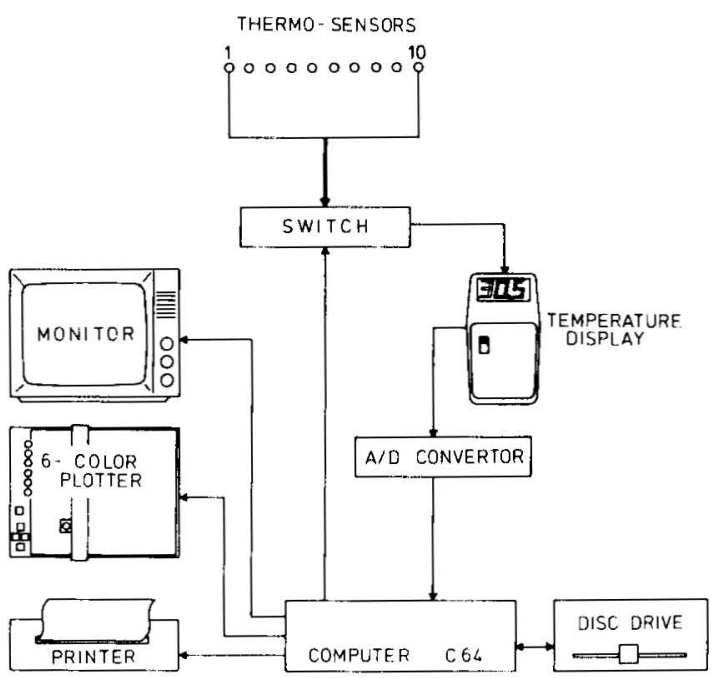

Figure 1. Fingertip thermometry system. $(A / D=$ analogue/ digital)

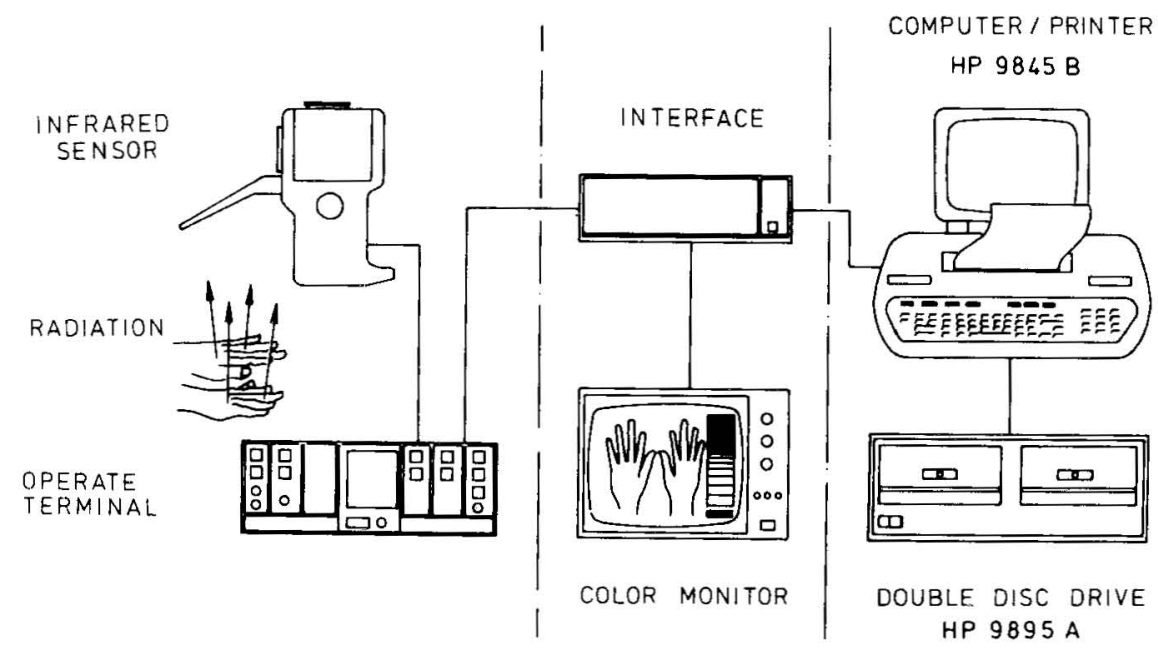

Figure 2. Infrared thermography system. 


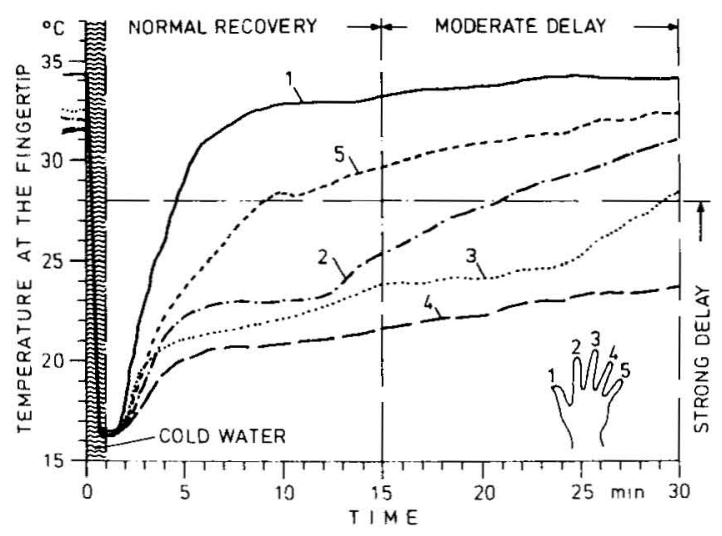

Figure 3. Examples of different recovery reactions after cold provocation in five fingers of one hand.

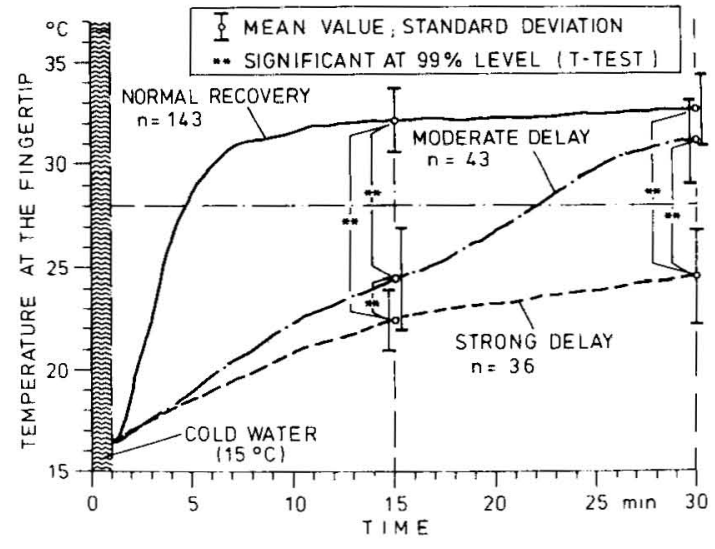

Figure 4. Recovery of fingertip temperature during a cold prov. ocation test (mean, standard deviation, and significance for three groups with different types of recovery) -222 subjects.

Moderate delay: Fingertip skin temperature of $\geq 28^{\circ} \mathrm{C}$ within 16-30 min

Strong delay: Fingertip skin temperature of $<28^{\circ} \mathrm{C}$ at end of test $(30 \mathrm{~min})$

A more differentiated assessment did not seem justified. Both thermometric methods may be used for the evaluation of cold provocation tests.

For the initial evaluation of the reaction type of a subject the "worst" of the 10 fingers was decisive. If all 10 fingers showed normal recovery, the subject would be evaluated as normal. If one or more fingers showed moderate delay or strong delay, the subject was placed in the moderate-delay or strong-delay group, respectively. Figure 4 shows the summarized assessment of the skin temperature recovery of 220 subjects during the cold provocation test. It may be seen that at $15 \mathrm{~min}$ the mean skin temperature of the worst finger increased to $32.1^{\circ} \mathrm{C}$ in the normal group, to $24.5^{\circ} \mathrm{C}$ in the moderate-delay group, and only to $22.4^{\circ} \mathrm{C}$ in the strong-delay group. All group differences were statistically significant. At the end of the test (30th minute) the skin temperature of the normal group reached $32.7^{\circ} \mathrm{C}$, that of the moderate-delay group $31.1^{\circ} \mathrm{C}$, and that of the strong delay group $24.7^{\circ} \mathrm{C}$. The differences between the normal and strong-delay groups and between the moderate-delay and strong-delay groups were statistically significant.

Within the normal group there was no statistically significant increase in skin temperature between the 15 th and 30th minutes, ie, the recovery process was complete already at the 15 th minute. The corresponding increase in the moderate-delay group was statistically significant, signifying that the main recovery took

Figure 5. Skin temperature at four points along the finger for three types of rewarming at 14 and $30 \mathrm{~min}$. (RP = reference points) 
place after the 15 th minute. For subjects of the strongdelay group however, there was only a very small increase in skin temperature. This finding demonstrates a very low level of peripheral circulation. On the basis of the statistical analysis made, it may be concluded that an assessment according to three different reaction types (normal, moderate delay, strong delay) is justified.

In a recent study by Steeger (personal communication) on intraindividual variation in skin temperature reaction with eight repetitions of the cold provocation test on different days, a very high consistency (variation coefficient $<5 \%$ ) in temperature recovery was demonstrated for eight of nine healthy subjects. Variation in temperature reaction may reflect the different physiological types (acrohomoiothermal, acropoikilothermal) of Klüken (2) (1964). Further research on intraindividual variation between VWF patients in cold provocation tests is needed.

The kind of evaluation of cold provocation tests with fingertip thermometry or infrared thermography described in this report is based on temperature measurements from reference points of ten fingertips and, therefore, allows a comparison of temperature recovery between all the fingers of a subject. This procedure proved to be very important since, in many cases of VWF, the peripheral circulation disturbance only concerns single fingers, mostly exposed to vibration and static forces. On the contrary, Raynaud's phenomenon of constitutional origin generally shows delayed temperature recovery for all fingers. A cold provocation test of this kind therefore may be of differential diagnostic value.

Infrared thermography, however, gives additional information which may be used in a second step of assessment. With this method it is possible to evaluate circulation disturbances on the basis of spatial and temporal changes in finger skin temperature.

Figure 5 shows the mean skin temperature at four points along the fingers at 14 and $30 \mathrm{~min}$ of the test (corresponding to the 13th and 29th minutes after the end of the cold provocation). In the 1732 fingers assessed as normal, the temperature was high at distal points 2 and 3 . In the two proximal points ( 1 and 0 ), however, the temperature was $0.5-1.0^{\circ} \mathrm{C}$ lower at 14 min than at $30 \mathrm{~min}$. In the 257 fingers given a moderate-delay rating, the temperature distribution at
14 and 30 min was very different. At 14 min the temperature tended to be progressively lower from proximal to distal, whereas at $\mathbf{3 0} \mathrm{min}$ the opposite was observed. This finding reflects the course of rewarming, which starts distally. For the 231 fingers with a strongdelay rating, there was a clear decrease in skin temperature from proximal to distal at both 14 and $30 \mathrm{~min}$. The skin temperature of the fingertip was about $3^{\circ} \mathrm{C}$ lower than that of proximal point 0 as a result of strong peripheral vasoconstriction.

In summary, the following conclusions may be drawn:

1. Skin temperature must be measured on all 10 fingers during the cold provocation test to enable a comparison of temperature recovery. Observed finger differences in rewarming rate may also be of differential diagnostic value.

2. Fingertip thermometry and infrared thermography are both suitable for the testing of finger thermoregulation.

3. The temperature may be measured either on the volar or on the dorsal surface of the fingertip.

4. The following three types of temperature reaction may be recognized: normal recovery, moderate delay in recovery, and strong delay in recovery.

5. Temperature measurements on the fingertips are sufficient for most subjects. In advanced cases, measurements along the length of the finger with infrared thermography may yield more information on the development of disturbances in peripheral circulation.

6. Considering the expense and effects of both the two methods used in this study to measure skin temperature, fingertip thermometry may be used with advantage in occupational health examinations, and infrared thermography is suitable for special clinical work.

\section{References}

1. Dupuis, H. Untersuchung zu vibrationsbedingten Durchblutungsstörungen der Hände. Schriftenreihe des Hauptverbandes der Gewerblichen Berufsgenossenschaften, Sankt Augustin 1986.

2. Klüken, N. Regulationsmechanismen an den Akren. Bibl Anat 4 (1964) 227-233. 